\title{
A correção das estenoses traqueobrônquicas mediante o emprego de órteses*
}

\author{
Maurício Guid Saueressig ${ }^{1}$, Amarílio Vieira de Macedo Neto ${ }^{2}$, AleXandre Heitor Moreschi ${ }^{3}$, \\ Rogério Gastal XavieR ${ }^{4}$, Paulo Roberto Stefani Sanches ${ }^{5}$
}

O tratamento cirúrgico do paciente com estenose traqueobrônquica exige uma avaliação individualizada em razão da complexidade de sua origem, sendo a traqueoplastia considerada a modalidade ideal. As causas mais comuns de estenose são devidas à intubação traqueal $e$ ao desenvolvimento de neoplasias e estas condições são justamente as que mais se beneficiam com o tratamento endoscópico quando a correção cirúrgica não está indicada. Na atualidade, os meios endoscópicos incluem a aplicação de diversos tipos de laser e sondas de dilatação, habitualmente com o uso de broncoscópio rígido, e emprego de radioterapia e órteses, separadamente ou em associação. Basicamente, as órteses são de dois tipos: metálicas e de silicone. As metálicas têm sua indicação mais freqüente para os casos de traqueomalacia e estenoses por compressão neoplásica extrínseca. Nos casos de obstrução da via aérea por inflamação aguda, restrita à sua luz ou sem envolvimento mais profundo da parede traqueobrônquica, ou em presença de neoplasia endoluminal, é recomendável a órtese de silicone. Embora em algumas situações essas diversas formas de tratamento possam ser intercambiáveis, não costumam ser a regra. Portanto, a estratégia terapêutica visando à resolução mais eficaz da estenose traqueobrônquica baseia-se na seleção dos métodos empregados desde o

primeiro tratamento. (J Pneumol 2002;28(2):84-93)

\section{Therapeutic management of tracheobronchial stenosis with stent application}

The surgical treatment of patients with tracheobronchial stenosis requires individualized attention due to the complexity of its origin though tracheoplasty is considered to be the ideal procedure. The most common causes of stenosis are tracheal intubation and the development of neoplasias.

These are the very conditions that most benefit from endoscopic treatment when surgical correction is not indicated. Today, endoscopic procedures include the application of different kinds of laser and dilators usually delivered with a rigid bronchoscope, as well as radiotherapy and stents that may be used separately or associated. Basically, there are two types of stents, metal or silicone. The metallic ones are more frequently indicated in cases of tracheomalacia and extrinsic compressive stenosis. Silicone are more applicable in cases of tracheobronchial obstruction resultant from acute inflammation or endoluminal tumor. Although these methods can be used interchangeably in some cases, the best way to correct tracheobronchial stenosis is to correctly decide upon the treatment as early as the preliminary steps.

Descritores - Traqueal. Dispositivo de fixação ortopédica. Angioplastia.
Key words - Tracheal stenosis. Orthopedics fixation devices. Angioplasty.
* Trabalho realizado na Universidade Federal do Rio Grande do Sul, RS.

1. Aluno do Programa de Pós-Graduação em Medicina: Cirurgia.

2. Doutor; Professor Adjunto do Departamento de Cirurgia.

3. Mestre; Programa de Pós-Graduação em Medicina: Cirurgia.

4. Doutor; Professor Adjunto do Departamento de Medicina Interna.

\section{Mestre; Programa de Pós-Graduação em Engenharia.}

Endereço para correspondência - Dr. Maurício Guidi Saueressig, Rua Ramiro Barcelos, 2.171, apto. 21 - 90035-007 - Porto Alegre, RS. Tels. (51) 321-2101 ou 9969-9932; e-mail: mguidi@zaz.com.br

Recebido para publicação em 12/3/01. Aprovado, após revisão, em 12/12/01. 


\section{INTRODUÇÃO}

O tratamento cirúrgico e endoscópico das estenoses de vias aéreas é um problema difícil e desafiador, principalmente devido à complexidade relacionada às suas causas. Estas podem ser fixas ou dinâmicas, como a traqueomalacia. As fixas podem ser divididas em: infecciosas, isquêmicas (após transplante pulmonar, por exemplo), auto-imunes devido à granulomatose de Wegener, sarcoidose e amiloidose, traumáticas, inflamatórias e neoplásicas. As estenoses traumáticas e inflamatórias podem ser pós-operatórias, secundárias à intubação prolongada, inalação de fumaça ou vapor e a trauma externo fechado. A intubação prolongada é a principal causa de estenose laringotraqueal, sendo seguida pelas neoplasias ${ }^{(1)}$. É importante ressaltar que a estenose subglótica ou glótica é originada de problemas relacionados ao tubo orotraqueal $e$ seu diâmetro e geralmente acompanha a estenose da traquéia cervical ${ }^{(2)}$.

A estenose de vias aéreas inferiores traz sintomas desconfortáveis e característicos, como o estridor, pneumonia pós-obstrutiva e dispnéia, podendo ser confundida com outras doenças devido à sua raridade. Em relação à traqueomalacia, apresentam-se em $75 \%$ dos pacientes de um a três meses após a extubação ${ }^{(3)}$. Esses sintomas são muitas vezes insidiosos e o diagnóstico costuma ser feito somente quando a luz traqueal está reduzida em mais de $60 \%$.

As estenoses benignas de vias aéreas centrais são geralmente secundárias a trauma por intubação traqueal. Segundo Brichet et al. ${ }^{(3)}$, as estenoses ocorrem na proporção de um caso para cada 1.000 pacientes intubados; contudo, trabalhos como o de Forte ${ }^{(4)}$ apresentam prevalência maior de estenoses, sendo que essa taxa pode variar de 2,3 a $17 \%$. Sua causa está relacionada à pressão do balonete na parede da via aérea que, ao ultrapassar a pressão de perfusão capilar, induz a isquemia, inflamação, tecido de granulação e pericondrite na fase aguda $e$, em casos graves, fibrose e destruição completa da cartilagem traqueal, provocando a malacia ${ }^{(1,4)}$. Em 1993, Law et al. ${ }^{(5)}$ realizaram estudo prospectivo em 81 pacientes com intubação traqueal prolongada e foi observada incidência de $67 \%$ de lesões nas vias aéreas, sendo $14 \%$ de traqueoestenoses.

Ainda como exemplos de estenoses benignas podem ser citados os estreitamentos resultantes em locais de broncoplastias e irradiação.

As estenoses malignas são causadas geralmente por neoplasias primárias ou secundárias, em que o tratamento paliativo endoscópico é considerado fundamental.

Apesar das diferenças entre estenoses benignas e neoplásicas, há similaridades como o desenvolvimento de tecido de granulação e condromalacia verificada após tra- tamento com laser e dilatações das obstruções neoplásicas.

O cirúrgico é o tratamento de escolha para a maioria das estenoses benignas, isto é, para aquelas com extensão maior de $1 \mathrm{~cm}$ sem processo inflamatório agudo. Tem como princípios fundamentais promover a estabilidade da via aérea, preservar a fonação e o reflexo de tosse, assim como a deglutição, evitando a aspiração. As traqueoplastias, traqueostomias $e$ a colocação de órteses como o tubo de Montgomery são as principais modalidades cirúrgicas utilizadas ${ }^{(3,4,6)}$. Há situações que podem dificultar o tratamento cirúrgico, tornando-se contra-indicações relativas à traqueoplastia: por risco cirúrgico aumentado, principalmente em pacientes com doenças sistêmicas como diabetes e doença cardíaca, por lesão maior de $6 \mathrm{~cm}$ e pela recorrência de estenose após procedimento cirúrgico. Segundo Grillo(7), o principal fator de contra-indicação não é a doença em si, porém haver sido inadequado o primeiro manejo, cirúrgico ou endoscópico. A alternativa para esses casos complicados seria o tratamento endoscópico.

Nas últimas décadas, grandes avanços ocorreram no tratamento endoscópico das estenoses de vias aéreas. O tipo do tratamento dispensado depende da qualidade da lesão, do grau de estenose, extensão, causa, localização, urgência dos sintomas, material, técnica disponível e experiência do médico endoscopista. Dilatações, ressecções mecânicas, térmicas e fotoquímicas, radioterapia endoluminal, laser, braquiterapia, crioterapia, injeção intralesional de esteróide e órteses são os principais exemplos deste novo conjunto de alternativas terapêticas.

O tratamento endoscópico é o mais indicado em lesões com abundante tecido de granulação e exofíticas, que necessitam desobstrução urgente, sendo atingido geralmente o alívio imediato, além daquelas com reação inflamatória na fase madura com pequenos segmentos de fibrose endoluminal. Tem taxa de sucesso a médio $e$ longo prazo de $57 \%$, de acordo com estudo de Ossof et al. ${ }^{(8)}$, e $60 \%$ de acordo com Mehta et al. ${ }^{(9)}$, em até três aplicações. Tem eficácia limitada a $10 \%$ nas estenoses maiores que $1 \mathrm{~cm}$ de comprimento longitudinal, circunferenciais e com perda da integridade das cartilagens, ou seja, as classificadas como complexas ${ }^{(4,9,10)}$. Portanto, nos pacientes que forem considerados inoperáveis ou de alto risco cirúrgico, as únicas alternativas serão o uso do tubo de Montgomery ou de órteses.

A palavra stent ou órtese originou-se do nome do dentista britânico Charles R. Stent, que ainda no século XIX desenvolveu material de impressão dentária, e após começou a utilizá-lo como suporte de enxertos de pele ${ }^{(11)}$. Hoje, a palavra é usada para os métodos que mantêm a permeabilidade de estruturas tubulares. 
A aplicação do conceito atual para órtese traqueal iniciou-se em 1891 com Bond, que utilizou um tubo metálico em "T" para a correção de um caso de estenose traumática de traquéia. Brünings e Albrecht, em 1915, e Jackson, em 1951, consolidaram a aplicação broncoscópica de órteses rudimentares em borracha ou metal. Em 1965, Montgomery criou um tubo de silicone em forma de "T" para a via aérea e, em 1986, Wallace relatou o uso de órtese metálica auto-expansível. Em 1989, Dumon apresentou um modelo de tubo feito de silicone, especialmente projetado para a árvore traqueobrônquica, a ser implantado por via endoscópica e sem necessidade de traqueostomia ${ }^{(2,3,11,12)}$.

O objetivo da órtese é de manter o suporte cartilaginoso, eventualmente perdido pela via aérea, e de contrapor-se às forças compressivas da árvore traqueobrônquica que, ao contrário das órteses vasculares, não tem o suporte exercido pela pressão hidrostática do sangue. É geralmente um procedimento adicional às outras técnicas endoscópicas para manutenção da luz traqueobrônquica, além de permitir a fonação $e$ o acesso à luz traqueal, sem a necessidade de operação como com o tubo de Montgomery.

As principais indicações da órtese em estenoses são: compressões tumorais extrínsecas ou lesão em submuco- sa; tumor endobrônquico, em que a luz após uso de laser permanece menor que $50 \%$; crescimento rápido ou recorrência tumoral após aplicações de laser; perda do suporte cartilaginoso; obstrução por órtese esofágica ou com uso seqüencial de órtese esofágica e traqueal para fístula entre os dois órgãos; e nos pacientes inoperáveis com estenose benigna ou maligna ${ }^{(12)}$.

Brichet et al. ${ }^{(3)}$ sugerem um algoritmo prático para o manejo de estenoses benignas com o auxílio da órtese de Dumon, como é ilustrado na Figura 1.

\section{PRÉ-REQUISITOS FUNCIONAIS E ANATÔMICOS}

As órteses devem apresentar os seguintes requisitos:

- restabelecer e manter a luz da via aérea;

- não causar lesão cicatricial ou perfuração pela pressão localizada na mucosa;

- não deslocar-se após implantadas;

- não interferir com o transporte de muco, o que pode ser facilitado com a adição de revestimento de silicone ou outro polímero;

- ser biológica e imunologicamente inertes;

- e, finalmente, de fácil manipulação.

Atualmente, não se dispõe de órtese que apresente todos esses requisitos. Assim, a escolha resultará da indica-

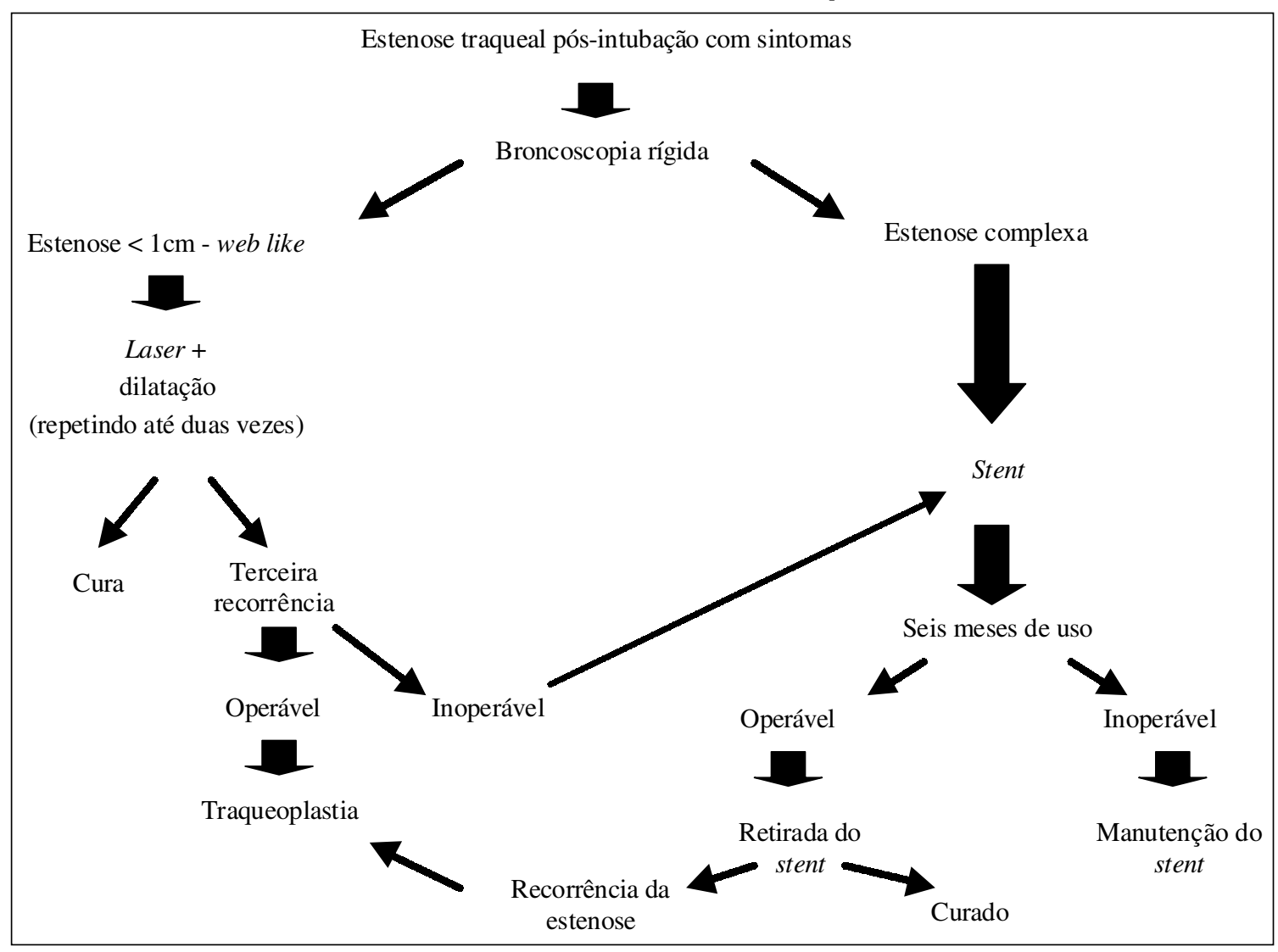

Figura 1 - Algoritmo do tratamento da estenose benigna traqueobrônquica segundo Brichet 
ção individual para cada caso e de avaliação entre os benefícios e riscos apresentados em cada modelo de órteses.

\section{TIPOS DE ÓRTESES}

São cinco os tipos básicos: tubo de silicone, metálico expansível por balão, metálico auto-expansível, híbrido e bioabsorvível, ilustrados na Figura 2. No Quadro 1, relaciona-se a experiência de diversos autores com as órteses, selecionados de acordo com os seguintes critérios: artigos pesquisados no Medline, a partir de 1990, com um número mínimo de 10 pacientes.

\section{Órtese de silicone}

O tubo em "T" de Montgomery (Fundação Adib Jatene, Brasil) foi o primeiro a ser construído com esse material em 1965(2), com diâmetros de 8, 10 e $12 \mathrm{~mm}$. O tubo produzido nos Estados Unidos tem diâmetros externos de 8 a $18 \mathrm{~mm}$. É indicado em estenoses subglóticas ou com comprometimento difuso e extenso da traquéia, nas quais as outras órteses não têm bons resultados, assim como nas falhas de tratamentos precedentes e como alternativa às traqueoplastias. Também é indicado por alguns autores quando da presença prévia de traqueosto$\mathrm{mia}^{(13,14)}$. Tem como vantagens a fácil limpeza de secreções espessas e excelente tolerância. Os bons resultados chegam a $85 \%{ }^{(4)}$. É a órtese mais utilizada no país(16).
A órtese de Dumon ${ }^{(17)}$ (Novatech, França), atualmente, é uma das de maior aceitação. Tem diâmetros externos de 10,12 e $13 \mathrm{~mm}$ e 14,15 e $16 \mathrm{~mm}$ e comprimento de 20, 30 e 40mm e 40, 50 e $60 \mathrm{~mm}$, respectivamente, para as aplicações brônquicas e traqueais. A introdução pelo broncoscópio rígido com auxílio de aplicador especial é simples, podendo ser controlada por fluoroscopia ou sob visualização direta com óptica adaptada. A presença de protrusões na forma de botões (studs) em sua superfície previne a migração e reduz o risco de isquemia da mucosa. Em casos de erro de colocação, deslocamento ou necessidade de retirada do expansor, esse é facilmente mobilizável com uma pinça de biópsia. Em caso de expansão insuficiente, utiliza-se um balão para moldá-lo, ou aguarda-se que se expanda gradualmente. Raramente obstrui ou permanece dobrado sobre si, pois o seu formato cilíndrico tende a promover distribuição das forças originárias da compressão extrínseca ${ }^{(14-18)}$.

Uma das principais, se não a mais freqüente complicação, é o deslocamento e a possível asfixia, sendo normalmente causada por: tamanho incorreto da órtese; pouca fixação devido à necrose de tumor após radioterapia; crescimento tumoral em uma das bordas da órtese, deslocando-a; diferenças na estimativa do diâmetro da via aérea, estando o paciente sob anestesia geral e após acordado; regressão de estenose inflamatória; dilatação progressiva da via aérea como processo de malacia; lesão curta (menor que $2,5 \mathrm{~cm}$ ), ou cônica, como nas lesões subglóticas;

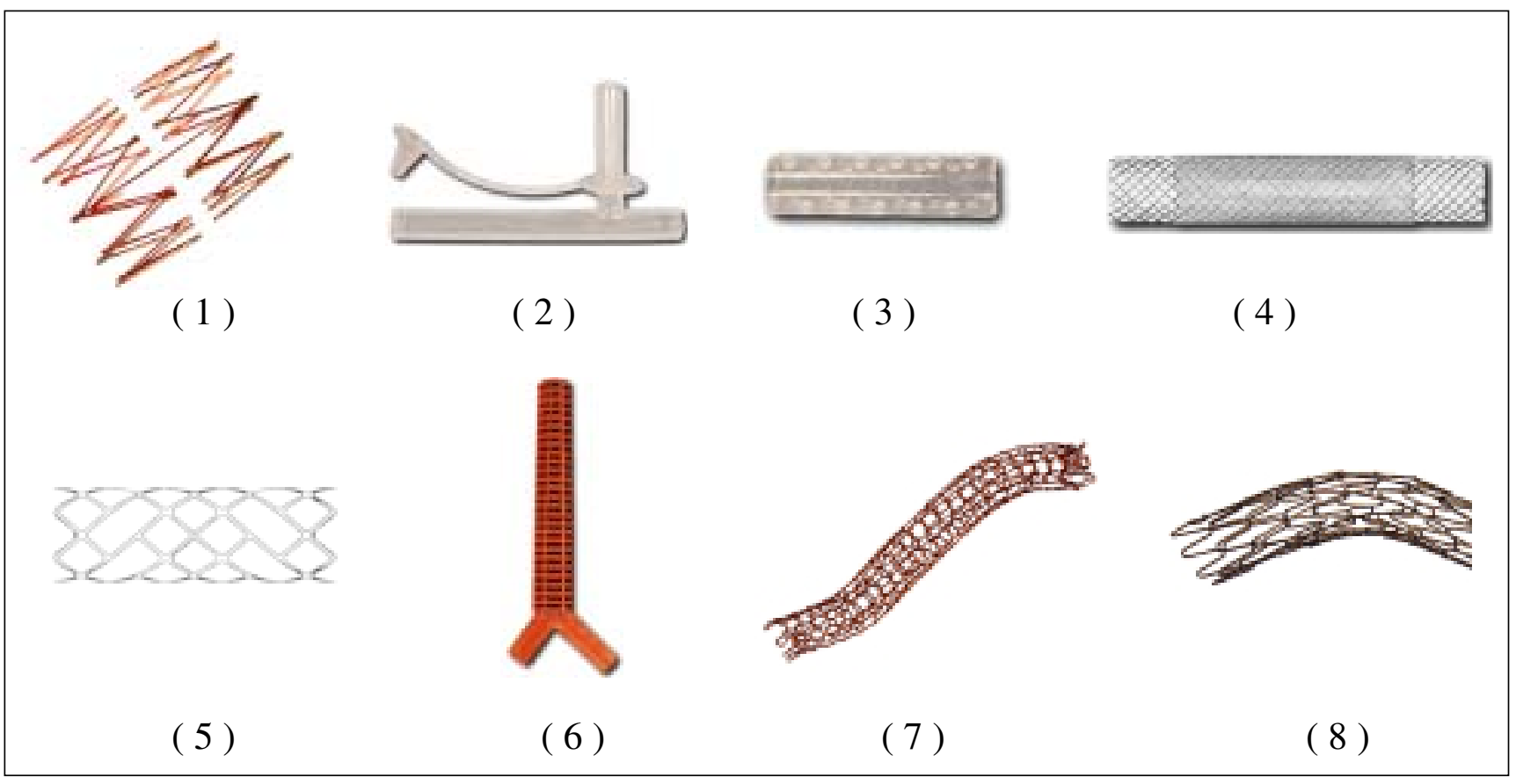

Figura 2 - Modelos de stents: 1) Gianturco, 2) Montgomery "T" tube, 3) Dumon, 4) Covered Wallstent, 5) Palmaz, 6) Freitag, 7) Strecker, 8) Cragg Nitinol. 
QUADRO 1

Experiência com órtese metálica e siliconizada: número de pacientes e complicações

\begin{tabular}{|c|c|c|c|c|c|c|c|}
\hline Autores*** & Stent & Casos & $\begin{array}{l}\text { Seguimento } \\
\text { médio (meses) }\end{array}$ & $\begin{array}{l}\text { Obstrução } \\
\text { por muco }\end{array}$ & Granuloma & Migração & Morte** \\
\hline $\begin{array}{l}\text { Forte et al., } \\
1993^{(16)}\end{array}$ & $\begin{array}{c}\text { Tubo de } \\
\text { Montgomery }\end{array}$ & $\begin{array}{c}69^{+} \\
31^{++}\end{array}$ & Até 96 & & & & $2 \%$ \\
\hline Júdice, 2001"\# & Gianturco & $\begin{array}{l}7 \mathrm{~N} \\
4 \mathrm{~B}\end{array}$ & 2 & NR & NR & $36 \%$ & NR \\
\hline $\begin{array}{l}\text { Puma et al., } \\
2000^{(49)}\end{array}$ & $\begin{array}{c}\text { Tubo de } \\
\text { Montgomery }\end{array}$ & $27 \mathrm{~B}$ & A té 83 & NR & NR & NR & NR \\
\hline Dumon, 1990(17) & Dumon & $\begin{array}{l}38 \mathrm{~N} \\
28 \mathrm{~B}\end{array}$ & 3 & $3 \%$ & $15 \%$ & $18 \%$ & NR \\
\hline $\begin{array}{l}\text { Bolliger et al., } \\
1993^{(46)}\end{array}$ & Dumon & $31 \mathrm{~N}$ & 2,5 & $6 \%$ & NR & $16 \%$ & $\begin{array}{c}1 \text { paciente } \\
3 \%\end{array}$ \\
\hline $\begin{array}{l}\text { Dumon et al., } \\
1996^{(18)}\end{array}$ & Dumon & $\begin{array}{l}698 \mathrm{~N} \\
360 \mathrm{~B}\end{array}$ & $\begin{array}{l}4 \mathrm{~N} \\
14 \mathrm{~B}\end{array}$ & $\begin{array}{l}1 \% \mathrm{~N}^{*} \\
7 \% \mathrm{~B}^{*}\end{array}$ & $\begin{array}{l}2 \% \mathrm{~N}^{*} \\
17 \% \mathrm{~B}^{*}\end{array}$ & $\begin{array}{l}6 \% *, \mathrm{~N} \\
15 \% *, \mathrm{~B}\end{array}$ & NR \\
\hline $\begin{array}{l}\text { Noppen et al., } \\
1996^{(50)}\end{array}$ & Screw-thread & $\begin{array}{l}5 \mathrm{~N} \\
5 \mathrm{~B}\end{array}$ & $\begin{array}{l}7 \mathrm{~B} \\
6 \mathrm{~N}\end{array}$ & & & NR & \\
\hline Brichet et al., 1999(3) & Dumon & $17 \mathrm{~B}$ & 6 & $29 \%$ & $11 \%$ & $11 \%$ & NR \\
\hline $\begin{array}{l}\text { Diaz-Jimenez et al., } \\
\text { 1994(14) }\end{array}$ & Dumon & $\begin{array}{l}60 \mathrm{~N} \\
30 \mathrm{~B}\end{array}$ & $\begin{array}{l}3 \mathrm{~N} \\
8 \mathrm{~B}\end{array}$ & NR & $\begin{array}{l}20 \%, B \\
6 \%, N\end{array}$ & $\begin{array}{l}22 \%, \mathrm{~B} \\
13 \%, \mathrm{~N}\end{array}$ & NR \\
\hline $\begin{array}{l}\text { M artínez-Ballarín } \\
\text { et al., } 1996^{(19)}\end{array}$ & Dumon & $63 \mathrm{~B}$ & 18 & $6,3 \%$ & $6,3 \%$ & $17,5 \%$ & NR \\
\hline $\begin{array}{l}\text { Cavaliere et al., } \\
1996^{(26)}\end{array}$ & Dumon & $306 \mathrm{~N}$ & 3 & $0,9 \%$ & $0,9 \%$ & $5 \%$ & NR \\
\hline $\begin{array}{l}\text { Noppen et al., } \\
1999^{(15)}\end{array}$ & $\begin{array}{c}\text { Dumon/ } \\
\text { Screw-thread }\end{array}$ & $\begin{array}{l}23 \mathrm{~N} \\
23 \mathrm{~B}\end{array}$ & até 72 & NR & $8 \% *$ & $\begin{array}{l}0 \% \mathrm{~N} \\
34 \% \mathrm{~B}\end{array}$ & NR \\
\hline $\begin{array}{l}\text { Nashef et al., } \\
1992^{(29)}\end{array}$ & Gianturco & $15 \mathrm{~B}$ & 13 & NR & $80 \%$ & NR & $\begin{array}{c}1 \text { paciente } \\
(6 \%)\end{array}$ \\
\hline $\begin{array}{l}\text { Rousseau et al., } \\
1993^{(23)}\end{array}$ & $\begin{array}{l}\text { Wallstent/ } \\
\text { Gianturco }\end{array}$ & $\begin{array}{l}50 \mathrm{~B} \\
5 \mathrm{~N}\end{array}$ & 10 & NR & NR & $\begin{array}{l}31 \% \text { com } \\
\text { Gianturco }\end{array}$ & $\begin{array}{c}1 \text { paciente } \\
(5 \%)\end{array}$ \\
\hline Sawada et al., 1993(51) & G ianturco & $14 \mathrm{~N}$ & 5,4 & NR & NR & NR & NR \\
\hline Carrasco et al., 1994(24) & 4) Gianturco & $36 \mathrm{~N}$ & 1,5 & NR & $5 \%$ & NR & NR \\
\hline Becker, 1995(45) & Nitinol/Strecker & $\begin{array}{l}59 \mathrm{~N} \\
36 \mathrm{~B}\end{array}$ & $\begin{array}{c}\text { 24, com o } \\
\text { nitinol em N } \\
6, B\end{array}$ & NR & NR & NR & NR \\
\hline $\begin{array}{l}\text { Perrone e Downey, } \\
1995^{(22)}\end{array}$ & Gianturco & $\begin{array}{l}9 \mathrm{~N} \\
6 \mathrm{~B}\end{array}$ & $\begin{array}{c}\text { até } 5, \mathrm{~N} \\
4, \mathrm{~B}\end{array}$ & NR & NR & NR & NR \\
\hline Shah et al., 1995(52) & Gianturco & $\begin{array}{c}23 \mathrm{~N} \\
4 \mathrm{~B}\end{array}$ & 8 & NR & $7 \%$ & $3 \%$ & NR \\
\hline $\begin{array}{l}\text { Bolliger et al., } \\
1996^{(25)}\end{array}$ & Wallstent recoberto & $27 \mathrm{~N}$ & 2 & $18 \%$ & $15 \%$ & $22 \%$ & NR \\
\hline $\begin{array}{l}\text { Monnier et al., } \\
1996^{(53)}\end{array}$ & Wallstent recoberto & $40 \mathrm{~N}$ & 8 & $38 \%$ & $10 \%$ & $12 \%$ & NR \\
\hline $\begin{array}{l}\text { Hauck et al., } \\
1997^{(21)}\end{array}$ & Nitinol/Strecker & $51 \mathrm{~N}$ & 4 & $39 \%$ & $1 \%$ & NR & NR \\
\hline \multicolumn{8}{|c|}{$\begin{array}{l}\text { * Percentagem calculada a partir do número de complicações/número de órteses colocadas; os demais foram calculado } \\
\text { pacientes do estudo. } \\
\text { Legenda: } \mathrm{N}=\text { neoplasia, B = benigno, NR: não relatado } \\
\text { ** Relacionada diretamente à órtese. } \\
\text { *** Trabalhos científicos selecionados através de pesquisa no Medline, indexados a partir do ano de 1990, como série de } \\
\text { \# Comunicação pessoal } \\
\text { + Estenose pós-intubação } \\
\text { ++ Estenoses com diversas etiologias: tuberculose, neoplasia, trauma, congênita, condrite, blastomicose, idiopática. }\end{array}$} \\
\hline
\end{tabular}


mucosa intacta, sem sinais de inflamação ou tumor $e$, finalmente, pelo fato de um órgão ductal estenosado ter diâmetros variados, provocando pressões desiguais ao longo do tubo ${ }^{(18)}$.

Outras eventuais desvantagens da órtese de silicone são: obstrução do orifício do brônquio lobar quando utilizado em bronco fonte, a maior espessura da parede em relação ao diâmetro interno, pouca flexibilidade às irregularidades anatômicas, alteração da fisiologia mucociliar e aparecimento de granulomas.

A órtese de Dumon tem a mesma tolerância quanto a do tubo de Montgomery. Bem posicionada, poderá ficar por longos períodos no local, usualmente sendo mantida por 18 meses ou mais para diminuir a probabilidade de recorrência. Esta pode alcançar $45 \%$, após um ano de permanência nas estenoses inflamatórias, de acordo com Dumon e Diaz-Jimenez ${ }^{(14,17-19)}$.

Assim, é a órtese de escolha nas estenoses inflamatórias traqueais e na presença de tumor, principalmente quando endobrônquico. Em estenoses neoplásicas, pode apresentar menor número de complicações se comparadas com as inflamatórias, talvez porque a resposta tecidual é menor e a fixação mais segura, principalmente, se levada em conta a compressão tumoral extrínseca(14-18).

\section{Órtese metálica expansível com balão}

Os modelos mais conhecidos são o de Palmaz (Cordis Corp; Johnson \& Johnson Interventional Systems Co; EUA), com comprimentos de 10 a 40mm e diâmetros de 8 a $12 \mathrm{~mm}$, constituído de aço e que apresenta fendas longitudinais em retângulo, e o de Strecker, formado por uma rede entrelaçada de fios de tântalo. Ambos de tamanho inicial reduzido, o que possibilita a passagem com facilidade em estenoses graves e podem ser introduzidos através de broncoscópio flexível sob anestesia local, o que é vantajoso, e dilatados na via aérea com auxílio de um balão expansível do tipo Gruenzig. A colocação deve ser precisa, pois deslocamentos subseqüentes podem destruir a estrutura metálica e lesionar a mucosa traqueal(20).

O de Palmaz pode ser recoberto pelo epitélio em toda a sua luz, integrando-se à parede brônquica. Entretanto, como foi originalmente projetado para utilização endovascular, podem acontecer problemas de colapso, migração ou reestenose pela inexistência da pressão hidrostática exercida pelo fluxo sanguíneo. Os melhores resultados com esse tipo de órtese foram em estenoses com presença de grande quantidade de tecido rígido de granulação, como nas subglóticas. Em estudos experimentais, houve incidência de tecido de granulação em até $85 \%$ dos pacientes, mas sendo mantida efetivamente a patência da luz traqueal ${ }^{(20,21)}$.

O de Strecker dispõe de boa flexibilidade, no entanto, com possibilidade de sofrer deformações mecânicas ou de, por sua rigidez, manter o diâmetro inicial mesmo após a regressão da lesão estenosante, provocando deslocamento $^{(21)}$. É útil em pequenas estenoses, como de brônquios segmentares, por apresentar comprimentos de 20 a $40 \mathrm{~mm}$.

\section{Órtese auto-expansível}

É aplicada através de um introdutor especial e expande-se quando liberada, promovendo a dilatação da parede traqueal. Os principais modelos confeccionados de aço são o de Gianturco (William Cook, Dinamarca) e o Wallstent (Schneider, Suíça).

Tanto a órtese de Gianturco como o Wallstent não podem ser removidos sem destruir a própria estrutura, devido à forte ancoragem após a expansão na luz brônquica, o que seria a maior desvantagem. Perrone e Downey ${ }^{(22)}$ desenvolveram expansores com fios de aço unidos por soldagem, que documentaram para aplicação temporária e retirada por via endoscópica de forma segura em um paciente.

Em relação às suas dimensões, a de Gianturco tem diâmetros em posição de relaxamento variando de 1,5 a $3 \mathrm{~cm}$ e comprimentos de 2 a $2,5 \mathrm{~cm}$, Entretanto, o Wallstent possui diâmetros de 10,12, 14 e $16 \mathrm{~mm}$ com comprimentos de 2,5 a $7 \mathrm{~cm}$.

Ao contrário dos similares, o Wallstent ${ }^{(13)}$ encurta-se em até $40 \%$ na aplicação, o que pode trazer dificuldade na escolha inicial quanto ao tamanho, mas apresenta revestimento que pode evitar o crescimento tumoral entre suas malhas.

Os expansores metálicos, ao contrário das órteses de silicone, devido à presença de fenestrações, não interferem com a função ciliar e permitem o crescimento de novo epitélio respiratório, podendo ser colocados, além da usual aplicação traqueal, nos brônquios lobares sem haver bloqueio dos seus orifícios segmentares, mas não previnem o crescimento de tecido inflamatório ou neoplásico para o interior da órtese $e^{(23)}$.

O manejo usual do crescimento tumoral na luz do expansor baseia-se na utilização de radiação endoluminal, laser, broncoscópio rígido e pinças de biópsia com resultados satisfatórios sem necessidade de troca pela órtese recoberta ${ }^{(21-24)}$. Entretanto, sendo indicada a colocação de uma órtese com cobertura, esta pode ser de teflon, dacron, silicone ou poliuretano, sendo especialmente útil no tratamento de fístulas esofágicas e para cavidade torácica associada a luz normal de via aérea, estando contraindicada a órtese de Dumon, segundo Bolliger et al. ${ }^{(25)}$, como também em casos de estenose neoplásica com luz irregular da via aérea, conforme Cavaliere et al. ${ }^{(26)}$. Porém, há poucos estudos prospectivos com resultados do uso desses expansores, e, mesmo com cobertura, não há 
prevenção absoluta do crescimento de tumor na luz da órtese (13,21-23,27,28). $^{2}$

Ao contrário do grupo de órteses anteriores, não colapsam com facilidade devido à força de expansão $e$ ao contínuo contato com as paredes brônquicas, com formação de aderências. Também têm como vantagem a colocação em pequenos cateteres de introdução, com diâmetros de até $3 \mathrm{~mm}$, podendo passar por intensas estenoses e naquelas de formato cônico. Essa força de expansão descrita é relacionada ao diâmetro, número de torções e espessura do fio de aço utilizado e pode aumentar rapidamente sob grandes forças compressivas, como pela tosse. Em geral, os expansores metálicos apresentam maior força radial e flexibilidade, além da alta relação entre diâmetro interno/externo, oferecendo grande patência de sua luz quando comparados com os de silicone.

Como desvantagens, apresentam maior tendência à formação de tecido de granulação, principalmente quando usados na vigência de processo inflamatório agudo, além da dificuldade em sua retirada, apenas alcançada com laser e/ou destruição de sua estrutura ${ }^{(23,29)}$. Há relatos de perfurações, fístulas e até de morte associada a esse tipo de órtese, principalmente com a de Gianturco, como mostrado no estudo de Rousseau et al. ${ }^{(23)}$, que tiveram $31 \%$ de migração e/ou ruptura da estrutura metálica, levando à obstrução ou perfuração. A fim de reduzir essas complicações, é crucial a escolha adequada das dimensões da órtese, assim como a presença de setas de ancoragem (studs) em sua estrutura ${ }^{(24)}$.

O expansor de nitinol (MinTec, França), também conhecido como Ultraflex, é constituído por liga de níquel $e$ titânio, sendo mais flexível que os anteriores. Com isso, apresenta mais similaridades mecânicas com a cartilagem traqueal. Exerce menor pressão sobre a parede brônquica e acarreta menor dano à mucosa ${ }^{(30)}$. Tem diâmetros externos de 14 a $20 \mathrm{~mm}$ e de 8 a $14 \mathrm{~mm}$ e comprimento variando de 40 e $80 \mathrm{~mm}$ e de 20 a $40 \mathrm{~mm}$, respectivamente, para a aplicação traqueal e brônquica. Embora de difícil visualização à fluoroscopia, tem mais possibilidades de ser reposicionado ou removido, além de apresentar potencial de expansão adicional, se houver regressão da estenose $^{(13,21,31,32)}$. A sua expansão é baseada no efeito denominado thermal-triggered shape memory: mediante aquecimento em altas temperaturas, a sua liga metálica especial é capaz de memorizar o formato helicoidal. Após aplicação na estenose, com o calor corporal é levado ao formato original, característica do Ultraflex, cuja plasticidade é de grande utilização em deiscências ou estenoses pós-operatórias com desvios da luz.

A órtese de nitinol e o Wallstent apresentam ainda modelos com cobertura, de fácil aplicação, mostram força radial adequada, boa flexibilidade e seriam os melhores expansores dessa categoria ${ }^{(13)}$.

\section{Órtese híbrida}

Este tipo de órtese incorpora metal e materiais poliméricos em sua construção, o modelo mais conhecido sendo o de Freitag (Rüsch AG, Alemanha), que possui corpo de silicone reforçado com anéis de metal. Apresenta formato em "Y" e diâmetros de 11, 13 e 15mm, com comprimento variável. Sua inserção é mais difícil, além de ser excessivamente rígido, porém é útil para estenoses próximas à carina ${ }^{(33)}$.

Entre as novas órteses desse tipo destacam-se: o de Hood, que apresenta arcos em vez dos studs de Dumon; o de Reynders, mais rígido que os anteriores e em forma de rosca, sendo composto de uma malha de poliéster, fina camada de silicone e auto-expansível; e o Novastent, constituído de fina camada de silicone e pequenos arcos de nitinol(6).

\section{Órtese bioabsorvível}

Há modelos experimentais em brônquios usando vários materiais como o poli-L-lactídeo ${ }^{(32)}$. Teria como vantagem a menor reatividade da mucosa respiratória, com a manutenção das propriedades do expansor metálico por um tempo específico.

Essas órteses têm ainda a retirada facilitada, especialmente em estenoses benignas, pela aplicação de solução salina gelada, que provocaria o seu recolhimento ${ }^{(35)}$.

\section{Iniciativas nacionais}

Entre as iniciativas nacionais de desenvolvimento de órteses de silicone para traquéia e brônquios, destaca-se a proposta de Xavier et al. ${ }^{(36)}$, que realizaram uma alteração do modelo de Dumon pela colocação de saliências externas desencontradas, seguindo o padrão dos anéis cartilaginosos em vez dos botões (studs) do original, com o intuito de garantir distribuição mais uniforme de pressões e impedir danos à mucosa, mesmo utilizando densidades diferentes de silicone adequadas aos diversos graus de rigidez da estenose.

Conforme a experiência relatada por Xavier et al. ${ }^{(36)}$, o Hospital de Clínicas de Porto Alegre (HCPA) possui um projeto experimental que compreende o desenvolvimento das órteses de silicone HCPA-1 (silicone biocompatível, grau implantável) e de metal (aço inox 316L). O HCPA-1 possui 4,5 a $7 \mathrm{~cm}$ de comprimento, diâmetro de 1,2 a $1,7 \mathrm{~cm}$ e paredes com espessura de $1 \mathrm{a} 2 \mathrm{~mm}$. O metálico apresenta dimensões de 40 × $20 \mathrm{~mm}$.

A partir de abril de 1999, seis casos com estenose traqueobrônquica foram manejados mediante essas órteses, com a exceção do primeiro paciente, no qual foi colocada a órtese de Dumon.

A colocação do HCPA-1 foi feita com broncoscópio rígido através de aplicador próprio desenvolvido pelo Serviço de Engenharia Biomédica do HCPA. 
A órtese de silicone foi utilizada em cinco pacientes, com média de idade de 31 anos. A estenose da traquéia cervical desses pacientes foi do tipo inflamatório, em três em conseqüência de intubação traqueal prolongada e em dois pela traqueostomia. Todos, exceto um, foram manejados inicialmente com traqueoplastia, dilatações e aplicação de laser YAG (CLMD/Dual, Surgical Laser Technologies, EUA). Um paciente permaneceu com a órtese de Dumon por 24 meses, sem apresentar problemas, mas ao ser retirado apresentou recorrência da estenose, necessitando novas aplicações de laser YAG. No segundo paciente, após quatro meses de colocação, iniciou-se formação de granulomas na borda superior $e$ inferior da órtese, não manejável eficientemente com laser YAG, sendo preciso retirá-la para colocação de tubo de Montgomery. O terceiro e o quarto pacientes permaneceram com as órteses por menos de dois meses, ocorrendo migração $e$ a presença de granulomas nas bordas, necessitando também a troca pelo tubo de Montgomery. A quinta paciente está assintomática após sete meses da colocação da órtese, sem sinais de complicações. Entretanto, a órtese metálica auto-expansível foi utilizada sem sucesso em uma paciente de 50 anos com obstrução do bronco fonte esquerdo por neoplasia metastática de rim e, apesar do seu posicionamento adequado, o pulmão não apresentou a reexpansão esperada, pelo comprometimento avançado do tumor no parênquima pulmonar associado à linfangite carcinomatosa.

As complicações da órtese de silicone no modelo HCPA1 são semelhantes àquelas encontradas na literatura, sendo que as estenoses por intubação traqueal dos três pacientes citados tinham localização subglótica, ou seja, com maior risco de migração, explicando em parte o ocorrido. $\mathrm{O}$ atrito das extremidades da órtese com a mucosa pode ter provocado a perpetuação do processo inflamatório, originando os granulomas nas bordas, o que deve ser solucionado com o aperfeiçoamento desse protótipo.

Há diversos serviços de pneumologia e de cirurgia torácica que apresentam sólida experiência no tratamento de estenoses traqueobrônquicas, que pode ser exemplificada nos trabalhos publicados em nossa literatura ${ }^{(36-44)}$. Destacam-se entre esses artigos a grande experiência acumulada de Forte $e^{(38)}$ no manejo das doenças das vias aéreas inferiores e a série de casos de Filomeno et al. ${ }^{(40)}$, que relatam os bons resultados da utilização do tubo de Montgomery em pacientes que apresentavam estenoses laringotraqueais extensas ou pela falta de condições clínicas para cirurgia.

É preciso destacar a grande experiência do Prof. Dr. Luiz Felippe Júdice, da Universidade Federal Fluminense, com as órteses de nitinol, Wallstent, Hood. A última órtese foi a mais utilizada na sua casuística, melhorando os sintomas respiratórios de $64 \%$ dos pacientes (comunicação pessoal).

\section{CONSIDERAÇÕES FINAIS}

Apesar de todos os progressos obtidos nesta área, ainda não foi conseguida a órtese ideal. As disponíveis apresentam características estruturais e funcionais diferentes e a escolha deve basear-se na capacidade de resolver imediatamente os sintomas obstrutivos, mas também levar em conta o potencial de complicações, a capacidade de manter pérvia a luz traqueobrônquica, a facilidade de colocação e retirada e ainda de conforto para o paciente.

A indicação mais comum das órteses é a obstrução neoplásica, sendo considerável a melhora na qualidade de vida dos pacientes. Verifica-se também razoável melhora na sobrevida aos que respondem satisfatoriamente ao tratamento conjugado de órtese e braquiterapia, atingindo até $50 \%$ em dois anos ${ }^{(21,45)}$. Diaz-Jimenez et al. ${ }^{(14)}$, Hauck et al. ${ }^{(21)}$, Carrasco et al. ${ }^{(24)}$, Bolliger et al. ${ }^{(25,46)}$ confirmaram sobrevida média de três a quatro meses, pela associação de radioterapia convencional.

O expansor metálico é de colocação relativamente fácil, sendo apontado como tratamento definitivo da traqueomalacia, segundo Perrone e Downey ${ }^{(22)}$. Também há grande aplicação nas estenoses malignas inoperáveis com compressão extrínseca, fístulas tumorais e obstruções com componente fibrótico isolado ${ }^{(29)}$. Não estaria indicado quando há proliferação de tecido inflamatório ou neoplásico na mucosa respiratória.

Segundo Becker ${ }^{(30)}$, os expansores metálicos com cobertura e, principalmente, o de nitinol, são os mais promissores, por assemelhar-se fisiologicamente à via aérea, sendo ainda necessário o desenvolvimento de coberturas eficientes e aplicadores de fácil manuseio sob anestesia local.

As órteses atuais de silicone são mais seguras que as metálicas, pois apresentam comportamento linear de compressão em resposta a força exercida externamente e a distribuição de sua pressão sobre a mucosa é delicada $e$ uniforme. As metálicas têm comportamento mais complexo e exercem pressão mais localizada sobre a mucosa, com fricção sobre pontos críticos durante momentos de compressão sobre as vias aéreas e bordas menos suaves e mais agudas, que pelo alongamento longitudinal podem também ocasionar estenoses em diafragma, ou estenose simples para diferenciar da estenose complexa, em que há lesão de toda a espessura da parede $e^{(3)}$.

Entretanto, a utilização da órtese comparada com outras terapias paliativas é segura, pouco invasiva, não necessitando de longas internações e oferecendo alívio rápido e duradouro dos sintomas. Em geral, a aplicação mais freqüente é em tumores inoperáveis ou irressecá- 
veis que provocam compressões extrínsecas de grandes vias aéreas ${ }^{(47)}$.

A órtese de silicone é a alternativa terapêutica mais promissora nas estenoses inoperáveis, ou ainda como procedimento paliativo e/ou temporário para obstruções inflamatórias em evolução antes da operação, sendo que seu tempo de permanência não deve ser inferior a seis meses, segundo Dumon et al. ${ }^{(18)}$.

As vantagens da órtese metálica são a menor probabilidade de migração e asfixia, a possibilidade do uso em brônquios para lobos inferiores sem obstruir os superiores, maior diâmetro interno em relação ao externo $e$ força de expansão residual até atingir o diâmetro predeterminado, oferecendo adequada função ventilatória e de limpeza de secreções, maior capacidade de adaptar-se às curvas anatômicas da via aérea. Bolliger et al.(25) citam ainda a facilidade da órtese metálica em se adaptar a diâmetros variados de uma estenose, podendo ser usada naquelas de formato cônico.

Embora as complicações relacionadas às órteses sejam freqüentes, podem ser manejadas com o broncoscópio de forma rápida e eficaz pela observação cuidadosa quanto ao aparecimento dos seguintes sintomas: tosse aguda com ou sem dispnéia pela migração ou infecção, dispnéia lentamente progressiva pela formação de granulomas, $e$ insuficiência respiratória aguda por obstrução ou secreção.

Na ausência de sintomas é desnecessária a realização de broncoscopias flexíveis de rotina após a colocação das órteses de silicone ${ }^{(48)}$.

A aplicação de órteses deve ser feita com indicação precisa e, como as demais medidas citadas para o manejo das estenoses traqueobrônquicas, não está livre de complicações. Tais medidas usadas de maneira arbitrária podem transformar uma estenose ainda reparável cirurgicamente em uma obstrução longa, irreversível e irressecável.

\section{REFERÊNCIAS}

1. Courey MS. Airway obstruction: the problem and its causes. Otolaryngol Clin North Am 1995;28:673-83.

2. Tedde ML, Nakakubo S, Nakamura RK, Minamoto H. Broncoscopia terapêutica nas estenoses: dilatações e próteses. In: Fernandes ALG, Mendes ESPS, Terra Filho M, editores. Pneumologia - Atualização e reciclagem. São Paulo: Atheneu, 1999.

3. Brichet A, Verkindre C, Dupont J, Carlier ML, Darras J, Wurtz A, et al. Multidisciplinary approach to management of postintubation tracheal stenoses. Eur Respir J 1999;13:888-93.

4. Forte V. Ressecção da estenose traqueal pós-intubação com reconstrução da traquéia por anastomose laringo, crico ou traqueotraqueal: análise clínica e cirúrgica. [Tese Livre-Docência], São Paulo: Universidade Federal de São Paulo, Escola Paulista de Medicina, 1996;206p.

5. Law JH, Barnhart K, Rowlett W, de la Rocha O, Lowenberg S. Increased frequency of obstructive airway abnormalities with long-term tracheostomy. Chest 1993;104:136-8
6. Wanamaker JR, Eliachar I. An overview of treatment options for lower airway obstruction. Otolaryngol Clin North Am 1995;28:751-69.

7. Grillo, HC. Stents and sense. Ann Thorac Surg 2000;70:1142.

8. Ossoff RH, Tucker GF, Duncavage JA, Toohill RJ. Efficacy of bronchoscopic carbon dioxide laser surgery for benign strictures of the trachea. Laryngoscope 1985;95:1220-3.

9. Mehta AC, Lee FY, Cordasco EM, Kirby T, Eliachar I, De Boer G. Concentric tracheal and subglottic stenosis. Management using the $\mathrm{Nd}$ YAG laser for mucosal sparing followed by gentle dilatation. Chest 1993;104:673-7.

10. Bisson A, Bonnette P, el Kadi NB, Leroy M, Colchen A, Personne C, et al. Tracheal sleeve resection for iatrogenic stenoses (subglottic laryngeal and tracheal). J Thorac Cardiovasc Surg 1992;104:882-7.

11. Baugnée PE, Marquette $\mathrm{CH}$, Ramon P, Darras J, Wurtz A. Traitement endoscopique des sténoses trachéales post-intubation. À propos de 58 cas. Rev Mal Respir 1995;12:585-92.

12. Nesbitt JC, Carrasco CH. Expandable stents. Chest Surg Clin North Am 1996;6:305-28.

13. Rafanan AL, Mehta AC. Stenting of the tracheobronchial tree. Radiol Clin North Am 2000;38:395-408.

14. Diaz-Jimenez JP, Munoz EF, Ballarin JIM, Kovitz KL, Presas FM. Silicone stents in the management of obstructive tracheobronchial lesions: 2-year experience. J Bronchol 1994;1:15-8.

15. Noppen M, Meysman M, Claes I, D'Haese J, Vincken W. Screw-thread vs Dumon endoprosthesis in the management of tracheal stenosis. Chest 1999; 115:532-5

16. Forte V, Perfeito JAJ, Imaeda CJ, Mercúrio Netto S, Leão LEV. Utilização do tubo "T" de Montgomery nas doenças laringotraqueais. J Pneumol 1993;19(S1):12-3.

17. Dumon, JF. A dedicated tracheobronchial stent. Chest 1990;97:32832.

18. Dumon JF, Cavaliere S, Diaz-Jimenez JP, Vergnon JM, Venuta F, Dumon MC, et al. Seven-year experience with the Dumon prosthesis. J Bronchol 1996;3:6-10.

19. Martínez-Ballarín JI, Diaz-Jiménez JP, Castro MJ, Moya JA. Silicone stents in the management of benign tracheobronchial stenoses, tolerance and early results in 63 patients. Chest 1996;109:626-9.

20. Fraga JC, Filler RM, Forte V, Bahoric A, Smith C. Experimental trial of balloon-expandable, metallic Palmaz stent in the trachea. Arch Otolaryngol Head Neck Surg 1997;123:522-8.

21. Hauck RW, Lembeck RM, Emslander HP, Schömig A. Implantation of Accuflex and Strecker stents in malignant bronchial stenoses by flexible bronchoscopy. Chest 1997;112:134-44.

22. Perrone R, Downey D. A self-expanding antiskid model stent removable by the endoscopic route: its usefulness in airways. $\mathrm{J}$ Bronchol 1995;2:20-6.

23. Rousseau H, Dahan M, Lauque D, Carré P, Didier A, Bilbao I, et al. Self-expandable prostheses in the tracheobronchial tree. Radiology 1993;188:199-203.

24. Carrasco CH, Nesbitt JC, Charnsangavej C, Ryan B, Walsh GL, Yasumori $\mathrm{K}$, et al. Management of tracheal and bronchial stenoses with the Gianturco stent. Ann Thorac Surg 1994;58:1012-7.

25. Bolliger CT, Heitz M, Hauser R, Probst R, Perruchoud AP. An airway wallstent for the treatment of tracheobronchial malignancies. Thorax 1996;51:1127-9.

26. Cavaliere S, Venuta F, Foccoli P, Toninelli C, La Face B. Endoscopic treatment of malignant airway obstructions in 2,008 patients. Chest 1996;110:1536-42.

27. Uchida BT, Putnam JS, Rosch J. Modifications of Gianturco expandable wire stents. Am J Roentgenol 1988;150:1185-7.

28. Bolliger CT, Arnoux A, Oeggerli MV, Lukic G, Perruchoud AP. Covered wallstent insertion in a patient with conical tracheobronchial stenosis. J Bronchol 1995;2:215-8. 
29. Nashef SAM, Dromer C, Velly JF, Labrousse L, Couraud L. Expanding wire stents in benign tracheobronchial disease: indications and complications. Ann Thorac Surg 1992;54:937-40.

30. Becker $H$. Flexible versus rigid bronchoscopic placement of tracheobronchial prostheses (stents). J Bronchol 1995;2:252-6.

31. Shaffer JP, Allen JN. The use of expandable metal stents to facilitate extubation in patients with large airway obstruction. Chest 1998;114: 1378-82.

32. Schmassmann A, Meyenberger C, Knuchel J, Binek J, Lammer F, Kleiner B, et al. Self-expanding metal stents in malignant esophageal obstruction: a comparison between two stent types. Am J Gastroenterol 1997;92:400-6.

33. Heitz M, Bolliger CT. Tracheobronchial insertion of the dynamic (Rüsch) stent. J Bronchol 1997;4:250-4.

34. Korpela A, Aarnio P, Sariola H, Törmälä P, Harjula A. Bioabsorbable bronchial stent in an animal model. J Bronchol 1998;5:9-13.

35. Fan Z, Ning D, Chen L. Expandable thermal-shaped memory metal esophageal stents: experiences with a new nitinol stent in 129 patients. Gastrointest Endosc 1997;46:352-7.

36. Xavier RG, Sanches PRS, Macedo-Neto AV, Silva-Filho APF, Edelweiss MII, Duarte L, Kuhl G, Fraga JC. Report of a new silicone stentbronchoscope introducer studied in dogs. Chest 2000;118(Suppl):234S.

37. Maurício L, Marques A, Rosa CM, Meneses F, Costa MF, Queiroz MV Policondrite recidivante com traqueobroncomalácia grave: a propósito de um caso clínico raro. Rev Bras Reumatol 1997;37:113-7.

38. Forte $\mathrm{V}$. O que mudou no tratamento dos tumores da traquéia nas últimas duas décadas. Rev Bras Cir Cabeça Pescoço 1987;11:11-22.

39. Otaviano AG, Evora PR, Barbieri Neto J, Carneiro JJ, Sader AA. Prótese traqueal - Estudo experimental em cães. Rev Col Bras Cir 1983; 10:46-53.

40. Filomeno LTB, Jatene FB, Minamoto H, Rodrigues OR, Campos JRM. Utilização do tubo em "T" de silicone no tratamento de estenoses laringo-traqueais complexas. Rev Col Bras Cir 1987;14:18-26.

41. Kuhl IA. Laringologia prática ilustrada. 2a ed. Rio de Janeiro: Revinter, 1996.
42. Thomé R. Trauma e estenose da laringe e da traquéia cervical. In: Brandão, LG, Ferraz, AR, editors. Cirurgia da cabeça e pescoço. São Paulo: Rocca, 1989;421.

43. Tedde ML, Nakakubo S, Nakamura RK, Minamoto H. Broncoscopia terapêutica nas estenoses: dilatações e próteses. In: Fernandes ALG, Silva Mendes ESP, Terra Filho M, editores. Pneumologia, atualização e reciclagem. Volume III, Sociedade Paulista de Pneumologia e Tisiologia. São Paulo: Atheneu, 1999;48-55.

44. Gomes NH, Gastal OL. Endoscopia torácica. In: Pinto Filho DR, Cardoso PFG, Figueiredo Pinto JAL, Schneider A, editores. Manual de cirurgia torácica. Sociedade de Cirurgia Torácica do RS. Rio de Janeiro: Revinter, 2001;72-82.

45. Becker H. Stenting of the central airway. J Bronchol 1995;2:98-106

46. Bolliger CT, Probst R, Tschopp K, Solèr M, Perruchoud AP. Silicone stents in the management of inoperable tracheobronchial stenoses. Indications and limitations. Chest 1993;104:1653-9.

47. Witt C, Dinges S, Schmidt B, Ewert R, Budach V, Baumann G. Temporary tracheobronchial stenting in malignant stenosis. Eur $\mathrm{J}$ Cancer 1997;33:204-8.

48. Matsuo T, Colt HG. Evidence against routine scheduling of surveillance bronchoscopy after stent insertion. Chest 2000;118:1455-9.

49. Puma F, Ragusa M, Avenia N, Urbani M, Droghetti A, Daddi N, Daddi $G$. The role of silicone stents in the treatment of cicatricial tracheal stenoses. J Thorac Cardiovasc Surg 2000;120:1064-9.

50. Noppen M, Dhaese J, Meysman M, Monsieur I, Verhaeghe W, Vincken W. A new screw-thread tracheal endoprosthesis. J Bronchol 1996; 3:22-6

51. Sawada S, Tanigawa N, Kobayashi M, Furui S, Ohta Y. Malignant tracheobronchial obstructive lesions: treatment with Gianturco expandable metallic stents. Radiology 1993;188:205-8.

52. Shah R, Sabanathan S, Mearns AJ, Featherstone H. Self-expanding tracheobronchial stents in the management of major airway problems. J Cardiovasc Surg 1995;36:343-8.

53. Monnier P, Mudry A, Stanzel F, Haeussinger K, Markus H, Probst R, et al. The use of the covered wallstent for the palliative treatment of inoperable tracheobronchial cancers. A prospective, multicenter study. Chest 1996;110:1161-8. 